\title{
The use of biomarkers in the diagnostics of fungal infections in children with oncological and hematological diseases
}

\begin{abstract}
Background and purpose: Patients with hematological malignancies are at risk of fungal infections and require quick diagnostics infection complications. The following study aimed to evaluate the effectiveness and relevance of the use of biomarkers of procalcitonin (PCT), C-reactive protein (CRP), galactomannan (GM), and bis (methylthio) gliotoxin (BMGT) in the diagnosis of fungal infections in patients with oncological and hematological diseases.
\end{abstract}

Materials and methods: The prospective study was conducted at the Belarusian Research Center for Pediatric Oncology, Hematology, and Immunology from April 2015 to January 2020. The study included 66 children with malignant hematological diseases aged 1 to 17 years. Clinical, microbiological, and statistical methods were used in the study.

Results: In the case of fungemia in children with oncological and hematological diseases, the PCT level during the infectious episode was significantly lower than with bacterial infections of the bloodstream $(p=0.0063)$; and the CPR level in cases of fungal and bacterial infections did not differ significantly $(p=0.1719)$. Diagnostic study of GM in bronchoalveolar lavage had a high predictive value of a negative result $(91.7 \%)$. The method's sensitivity was higher than in the study of GM in serum ( $50 \%$ versus $0 \%)$. There was no correlation between serum BMGT levels as measured by HPLC and the presence of invasive aspergillosis in children.

Conclusion: An increase in CRP levels with normal PCT levels in immunocompromised children with clinical signs of bloodstream infection is indicative of a fungal etiology of the disease. Determination of the optical density index of galactomannan in the bronchoalveolar fluid is a sensitive marker for diagnosing invasive pulmonary aspergillosis in children. We cannot recommend BMGT for the diagnostics of invasive aspergillosis in children.
Volume 9 Issue 4 - 202 I

\section{Sviatlana Kandaurava, Michael Tchernovetski, Olga Aleinikova \\ Belarusian Research Center for Pediatric Oncology, Hematology} and Immunology, Republic of Belarus

Correspondence: Sviatlana Kandaurava, Belarusian Research Center for Pediatric Oncology, Hematology and Immunology, Borovlyany, Minsk region, Republic of Belarus,

Email kandaurava@oncology.by

Received: August 12,2021 | Published: August 31,2021

Keywords: biomarkers, infectious diseases, oncohematological diseases

\section{Introduction}

Mycoses, or infections caused by microscopic fungi, have become a serious problem over the past two decades, affecting the morbidity and mortality of immunocompromised patients. Difficulties in the diagnosis of invasive fungal infections (IFI) lie in the fact that diagnosis requires a comprehensive assessment of the research results obtained, the clinical condition of the patient and the presence of risk factors. The clinical manifestations of fungal infections are nonspecific and the final diagnosis depends on invasive procedures, which are not always feasible due to the severe condition of the patient and concomitant cytopenia. Early diagnosis of these life-threatening complications requires rapid, minimally invasive, highly sensitive and specific tests, since late diagnosis and delay in therapy are potential risk factors for hospital mortality.

In most cases, the diagnosis of a fungal infection remains tentative. It can be confirmed by a combination of positive results of histological, microbiological, serological, molecular biological studies of primary sterile or potentially sterile material and the presence of clinical and instrumental symptoms of lesions of one or another organ. ${ }^{2-4}$ Combinations of clinical, radiological data and serological determination of fungal antigens (mannan, galactomannan, $(1,3)-\beta-\mathrm{D}-$ glucan) are used. The concentration of these antigens depends on the degree of invasion of pathogens, ${ }^{5,6}$ however, the sensitivity and specificity may vary depending on the administration of other drugs (galactomannan test). ${ }^{7,8}$ In addition, positive serological test results are possible in the absence of infection (with colonization). Therefore, the determination of fungal antigens is associated with the possibility of obtaining false positive and false negative results, which reduces the significance of these tests and does not completely guarantee a timely diagnosis. Determination of fungi by PCR is a promising method, but at the moment it is not recommended for widespread use in clinical practice. Due to the difficulties in diagnosis, international criteria for proving invasive fungal infections have been developed, which include possible, probable and proven IFIs. ${ }^{9}$

a. Proven IFI: the patient has clinical signs of IFI and when examining biological material from a sterile locus, fungal structures are microscopically detected or fungal culture growth is obtained;

b. Probable IFI: the immunocompromised patient has clinical signs of infection, as well as the presence of fungal elements on microscopy or inoculation of a culture of the fungus from a potentially sterile locus or positive serological tests (for example, a positive galactomannan test for aspergillosis);

c. Possible IFI: the immunocompromised patient is at risk, has risk factors and clinical signs of infection, and all microbiological studies are negative or not carried out. 
In terms of specificity, the culture method remains the "gold standard" of laboratory diagnostics of IFI, but it is inferior in sensitivity to molecular biological methods. As a rule, yeast fungi are secreted in blood cultures, but the sensitivity of blood cultures for candidemia is only $63-83 \%{ }^{7}$ This is a slow and low-sensitivity method that is not suitable for early diagnosis. ${ }^{10,11}$ A false negative result of blood culture can be observed with the prophylactic use of antifungal agents or empirical therapy. ${ }^{12}$

Treatment for infections caused by bacterial or fungal microflora varies greatly. Unjustified and untimely therapy leads to the development of adverse events, toxicity, antibiotic resistance and an increased risk of death. All of the above necessitates early identification of the etiology of infectious complications in immunocompromised oncohematological patients. Among the common diagnostic methods, the most commonly used biomarkers are C-reactive protein (CRP) and procalcitonin (PCT). The level of CRP, as an acute phase protein, increases in many pathological conditions, and an increase in PCT is usually associated with bacterial infection. ${ }^{13}$ There are publications on the results of an increased level of CRP and a low level of PCT in adult hematological patients with fungal infection in the study of the etiology of febrile neutropenia. ${ }^{14}$

The determination of galactomannan in body fluids was identified in 2002 by the EORTC/MSG as a diagnostic criterion that was evaluated in value as equal to the isolation of fungi of the genus Aspergillus or microscopic detection of hyphae from potentially sterile loci. Galactomannan test, together with clinical and radiological studies, complements the diagnosis of IA. Determination of galactomannan in the blood serum of patients with oncological and hematological diseases indicates a probable IA. ${ }^{15}$ Galactomannan is a polysaccharide that is part of the cell wall of fungi of the genus Aspergillus, and is released during the growth of hyphae. Determination of GM is carried out by the enzyme-linked immunosorbent assay in blood serum and bronchoalveolar lavage (BAL) fluid, and can also be performed by a similar method in cerebrospinal fluid (CSF) and urine. ${ }^{16}$ This method is approved in Europe and in the USA. Other methods for detecting this antigen were not sensitive enough for use in diagnostics. ${ }^{17}$ Circulating GM can be detected on average 5-8 days (with a range of 1 to 27 days) before the first clinical manifestations, changes on chest radiographs or positive cultures of the fungus. In addition, the concentration of circulating galactomannan correlates with the degree of tissue invasion and can be used to monitor the effectiveness of antifungal therapy. A high level of serum galactomannan index correlates with the presence of foci in the lungs $(p=0.037)$ and mortality $(p=0.001){ }^{18}$

It should be noted the frequent appearance of false-positive results of galactomannan studies in newborns due to cross-reaction with Bifidobacterium bifidum, which are found in high concentration in the gastrointestinal tract of children of this age group, cross-reactions with Penicillium marneffei or Cryptococcus neoformans. In addition, the sensitivity and specificity of the galactomannan test may vary depending on the administration of other drugs and transfusions of blood components. ${ }^{8,19,20}$ The use of P-lactam antibacterial agents can give false positive test results, which can persist up to 5 days after discontinuation of antibiotic therapy. ${ }^{21}$ False positive results are often detected within 100 days after HSCT in patients with chemotherapyinduced mucositis or GVHD. ${ }^{22}$ During this period, bacteria carrying cross-reactive antigens can enter the bloodstream through damaged mucous membranes of the gastrointestinal tract. ${ }^{23}$

Many fungi contain galactomannans (or polysaccharides containing galactofuranose residues) in the cell wall. False positive galactomannan test results can be observed with infections caused by these organisms (cross-reactive antigens). These include filamentous Ascomycetes, which are characterized by similar clinical manifestations, and others (Penicillium, Histoplasma capsulatum) ${ }^{24-26}$ Another reason for false positive blood test results for galactomannan is food contamination with fungi of the genus Aspergillus or Penicillium. Some food products contain galactomannan in the form of food additives (for example, guar gum is a food additive included in the group of stabilizers, and emulsifiers). ${ }^{27}$

There is also evidence of false negative results against the background of antifungal medical prophylaxis and therapy. ${ }^{28,29} \mathrm{~A}$ negative test result for serum galactomannan does not exclude the presence of other fungal infections, in particular Mucormycosis ${ }^{15}$ (fungi of the genus Mucor do not contain specific components ((13)-P-D-glucan or galactomannan) in the cell wall). The criterion for evaluating the test for the determination of GM is a comparison with the threshold value of optical density. In multivariate analysis, the BAL galactomannan test showed higher sensitivity data compared to direct microscopy $(83.3 \%$ and $46.3 \%$, respectively). With an optical density index equal to 1.0 and higher, the analysis of GM in the BAL fluid had a higher sensitivity and predictive value of a negative result in comparison with PCR and the test for determining GM in blood serum. It is a useful adjunct in the diagnosis of probable invasive aspergillosis (IA). ${ }^{30}$

It is known that during the growth of hyphae, fungi produce toxins. It is assumed that suppression of host immunity by mycotoxins (secondary metabolites released by fungi) is one of the possible mechanisms underlying the disruption of the human immune system's resistance to fungal growth and, accordingly, the development of fungal diseases. ${ }^{31}$ These toxins include gliotoxin (GT) and bis(methylthio) gliotoxin (BMGT), which are formed during the growth of Aspergillus spp., Eurotium Chevalieri, Gliocladium fimbriatum, as well as Penicillium and Trichoderma spp. GT has been proposed as a diagnostic biomarker for IA. ${ }^{32}$ GT mediates immunosuppressive and proapoptotic effects that determine the virulence of fungi during hyphal growth. Most commonly, GT is produced by the Aspergillus fumigatus species, which is responsible for most cases of IA. Other (non-A.fumigatus) species (A.flavus, A.terreus, A.niger) can also produce GT. ${ }^{33-35}$ Fungi of the genus Candida are the most common cause of invasive fungal infections and do not produce GT. ${ }^{36}$ All these characteristics make GT useful as a specific biomarker of IA. However, GT is a highly reactive compound and is rapidly absorbed by tissues and circulating cells, making it difficult to detect in body fluids. ${ }^{37}$ Conversely, bis(methylthio)gliotoxin, a more stable inactive metabolite, is formed by blocking the disulfide bond present in GT by methylation. BMGT has been identified in several GT-producing fungi, namely A.fumigatus, Penicillium spp., T.virens, Gliocladium spp., C.gloeosporoides or Pseudallescheria spp. According to ${ }^{37}$ BMGT production was found in cell cultures of A. fumigatus, A. flavus, $A$. niger, and $A$. nidulans, but not $A$. terreus. This study also demonstrated that BMGT is produced by a higher percentage of $A$. fumigatus isolates than GT. BMGT is also found in the serum of more patients at risk of IA than GT. These results suggest that BMGT is a more reliable indicator of infection than GT. Although both compounds are readily recovered from human serum or plasma, only BMGT is retained in whole blood, indicating that BMGT may be a better marker for in vivo detection.

According to the literature, BMGT has a higher sensitivity and predictive value of a positive result than galactomannan, and a similar specificity and predictive value of a negative result. The results of the BMGT study are promising and suggest that BMGT and GM can be used in combination to diagnose invasive aspergillosis and 
to select antifungal therapy. In general, the determination of fungal antigens and toxins is associated with the possibility of obtaining false positive and false negative results, which reduces the significance of these diagnostic methods and does not completely guarantee a timely diagnosis. In connection with the problems listed above, it is necessary to study diagnostic biomarkers, as well as their combinations. ${ }^{31}$

\section{Materials and research methods}

The prospective study was conducted at the Belarusian Research Center for Pediatric Oncology, Hematology and Immunology from April 2015 to January 2020. The study included 66 children with malignant hematological diseases aged 1 to 17 years. The study used clinical, microbiological and statistical methods.

Galactomannan was determined in blood serum and bronchoalveolar fluid (lavage) by enzyme immunoassay (PLATELIATM test, Bio-Rad Laboratories, France). A positive result of determining the optical density index of serum galactomannan was considered a single level of 0.7 or higher or two consecutive results of 0.5 and higher; ${ }^{38}$ and galactomannan of bronchoalveolar fluid-1.0 and higher. ${ }^{9}$ A false positive result for the determination of galactomannan was considered the result of a study in which the optical density index of GM in the test sample was equal to or exceeded the established diagnostic value in the absence of isolation of Aspergillus micromycetes from clinically significant biological material (fungal infection was not microbiologically confirmed). A false negative result for the determination of galactomannan was considered the result of a study in which the optical density index of GM in the test sample was below the established diagnostic value when isolating Aspergillus micromycetes from clinically significant biological material (microbiologically confirmed fungal infection).

According to a study by M.P. Domingo et al. bis(methylthio) gliotoxin was determined in the serum of sick patients by thin layer chromatography with mass spectrometry (TLC-MS), ${ }^{37}$ without the appropriate equipment, in our study the determination of serum BMGT was performed using highly efficient chromatographymass spectrometry, which, in comparison with TLC-MS, has a higher sensitivity. Bis (methylthio) gliotoxin (Abcam) was used as standards. Acetonitrile, methanol and formic acid (LC-MC grade) were manufactured by Fisher Chemical. Pure plasma (anticoagulant lithium heparin) was obtained at the Center for Pediatric Oncology, Hematology and Immunology from blood samples from healthy employees and donors by centrifugation (12,500 rpm). It was decided to consider the presence of a peak with a retention time of 2.82 minutes as evidence of the presence of bis (methylthio) gliotoxin in the patient's blood. It was decided to consider any result of BMGT positive. The sensitivity threshold of the method is $0.01 \mathrm{mg} / 1$. Youden's index suggests a diagnostic value for BMGT of $2.17 \mathrm{mg} / \mathrm{L}$ with a sensitivity of $61.5 \%$ and a specificity of $93.6 \%{ }^{39}$

Procalcitonin was determined in blood serum using an immunofluorescence analyzer (Cobase 411, Roche, Switzerland; Elecsys BRAHMS PKT kits, Roche, Switzerland). A level $>0.5 \mathrm{ng} / \mathrm{ml}$ was considered a positive value. C-reactive protein was determined using a biochemical analyzer (Architect c8000, Abbott, USA; CRP Vario kits, Abbott, USA). A level $>1.0 \mathrm{mg} / \mathrm{dL}$ was considered a positive value.

\section{Statistical analysis}

The cumulative incidence of fungal infections was determined by the method of competing risks, statistical differences in the groups were assessed by the Gray test. To establish indicators of sensitivity, specificity, predictive value of positive and negative results, a contingency table was used..$^{40}$ The analysis of quantitative indicators included the calculation of the main indicators of descriptive statistics: median, $25 \%-75 \%$ percentile, minimum, maximum. To confirm the hypothesis about the presence of statistical differences between two independent samples, the nonparametric Mann-Whitney test was used. For the graphical presentation of the quartile-median distribution of quantitative data, a range chart was used. The $\chi^{2}$ test was used to compare categorical data in the groups. All statistical differences were considered significant at $\mathrm{p}<0.05$.

\section{Results}

Procalcitonin and C-reactive protein in the differential diagnosis of fungal and bacterial infections. The PCT and CRP study included 17 patients with oncological and hematological diseases at the age from 1 to 17 years. All patients had laboratory-confirmed infectious episodes of bacteremia or fungemia. The blood was regarded as a sterile locus. Cases of fungemia belonged to the category of proven invasive fungal infection. ${ }^{9}$ The study included cases of bacterial bloodstream infection $(\mathrm{n}=10)$ caused by gram-negative bacteria: Klebsiella pneumoniae,Escherichia coli, Enterobacter cloacae and gram-positive bacteria Staphylococcus aureus. In cases of fungemia $(\mathrm{n}=7)$, pathogens were represented by yeast (Candida albicans and non-albicans) and molds (Fusarium spp.), Table 1.

Table I Results of detecting pathogens of fungal and bacterial infections of the bloodstream in the comparison groups

\begin{tabular}{|c|c|c|c|c|}
\hline \multirow[t]{2}{*}{ Characteristic } & \multicolumn{2}{|c|}{ Cases of fungemia } & \multicolumn{2}{|c|}{ Cases of bacteremia } \\
\hline & $\mathrm{n}$ & $\%$ & $\mathrm{n}$ & $\%$ \\
\hline \multicolumn{5}{|l|}{ Infectious episodes } \\
\hline & 7 & 100 & 10 & 100 \\
\hline $\begin{array}{l}\text { Gram-negative bacteria (K. pneumoniae } n=6, \text { E. coli } \\
n=2 \text {, E. cloacae } n=I)\end{array}$ & - & - & 9 & 90 \\
\hline Gram-positive bacteria (Staph.aureus $\mathrm{n}=\mathrm{I}$ ) & - & - & 1 & 10 \\
\hline $\begin{array}{l}\text { Yeasts (C. albicans } n=2 \text {; Candida non-albicans: } C \text {. } \\
\text { parapsilosis } n=I, C \text {. lipolytica } n=I, C \text {. tropicalis } n= \\
\text { I) }\end{array}$ & 5 & 71 & - & - \\
\hline Mold fungi (F.solani $\mathrm{n}=\mathrm{I}$, Fusarium spp., $\mathrm{n}=\mathrm{I}$ ) & 2 & 29 & - & - \\
\hline
\end{tabular}


Patients with viral or mixed infection were excluded from the study. All patients underwent PCT and CRP measurements (1 or more times) throughout the infectious episode. The indicators were evaluated in the interval of 7 days before and 14 days after the day of detection of a positive blood culture. Results are presented as median maximum values $(25 \%-75 \%)$.

Analysis of these maximum PCT values during an infectious episode differed significantly in infections caused by fungal and bacterial pathogens. The median maximum PCT values for fungal infections was $0.38 \mathrm{ng} / \mathrm{ml}(0.27-1.53)$, with bacterial infections-17.46 $\mathrm{ng} / \mathrm{ml}$ (3.59-45.01), $\mathrm{p}=0.0063$. With fungal infections of the bloodstream $(n=7)$, the maximum PCT level during the infectious episode was significantly lower than with bacterial infections of the bloodstream $(\mathrm{n}=10)$, Figure 1.

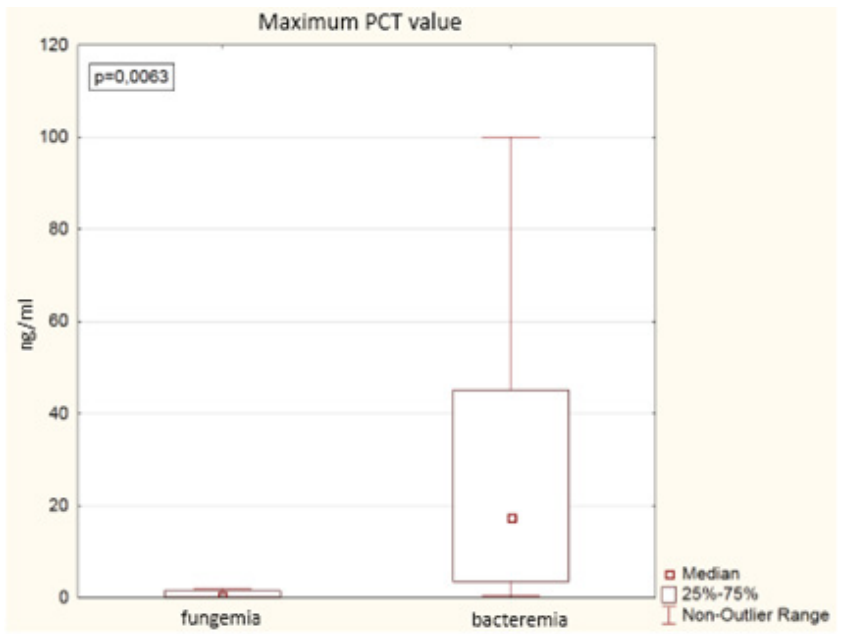

Figure I Results of PCT determination in children with fungemia $(n=7)$ and bacteremia $(n=10)$.

When comparing the maximum CRP values for an infectious episode in children, there was no significant difference in levels in infections caused by fungal or bacterial pathogens. The median of the maximum CRP values for fungal infections is $12.68 \mathrm{mg} / \mathrm{dL}(8.5-$ 19.12), for bacterial-17.53 mg/dL (12.89-24.9), p=0.1719, Figure 2 .

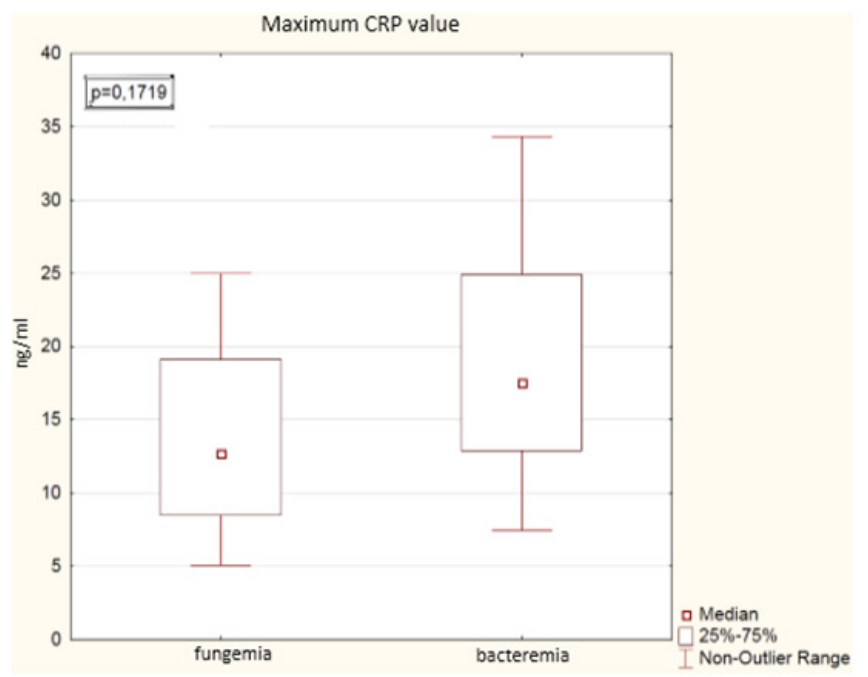

Figure 2 Determination of CRP in children with fungemia $(n=7)$ and bacteremia $(n=10)$.

\section{Evaluation of the effectiveness of methodologies for serological diagnosis of invasive aspergillosis (IA)}

Determination of galactomannan was carried out in the presence of signs of lower respiratory tract infection (focal-infiltrative changes in the lungs) in patients according to imaging studies (CT, X-ray).

We examined 33 patients aged 1 to 17 years (median age was 9 years), each of whom had focal-infiltrative changes in the lungs, which persisted despite the antibiotic therapy with broad-spectrum agents. In $9(27 \%)$ of these patients, decay cavities were visualized. Of the 33 examined, $6(18 \%)$ patients were allo-HSCT recipients. All patients underwent bronchoalveolar lavage with examination of GM in the obtained fluid.

Aspergillus infection was considered confirmed by microscopic detection of characteristic fungal structures (microscopic examination) or microbiological isolation of micromycetes (culture) from bronchoalveolar lavage material, biopsy or autopsy material of lung tissue of patients included in the study.

The fluid obtained during bronchoalveolar lavage was examined microbiologically and serologically (determination of galactomannan). In $29(87.9 \%)$ of 33 analyzed patients, the study of galactomannan in blood serum was carried out simultaneously with BAL.

In $9(27.3 \%)$ patients out of 33 analyzed, the result of galactomannan determination in BAL fluid was positive (optical density index $>1.0$ ). In 2 cases out of 9 positive results, Aspergillus infection was confirmed microbiologically (pathogens: Aspergillus fumigatus, Aspergillus niger). In 2 cases out of 24 negative results, Aspergillus infection was confirmed microbiologically (pathogens: Aspergillus flavus, Aspergillus spp.- without species identification).

In 29 out of 33 analyzed patients, serum GM was determined. In 3 cases, the result was positive. There were no cases of microbiologically confirmed aspergillosis among these 3 patients. Aspergillus infection was confirmed microbiologically in 4 cases out of 26 negative results for the determination of serum GM.

Aspergillus infection was accompanied by fever, cough, chest pain, wheezing in the lungs on auscultation, and hemoptysis. One patient was fatal due to pulmonary hemorrhage. One patient had a partial response to the ongoing antifungal therapy in the form of a decrease in focal changes in the lungs and an almost complete disappearance of the clinical symptoms of the disease, but died from other causes. Two patients have recovered.

The correspondence between a positive result of GM determination in blood (serum) and bronchoalveolar fluid was observed in one patient (in 1 case). Aspergillus infection in this case was not confirmed microbiologically.

Microscopic examination of the native material did not give positive results. All cases of invasive aspergillosis $(n=4)$ were identified by culture (Table 2).

By microbiological examination of the BAL material and biopsy or autopsy material of the lung tissue of patients, 4 (12\%) patients were found to have IFI (Table 2). In the GM study group in BAL ( $\mathrm{n}=33$ ), 7 cases of false-positive, 2 cases of false-negative results, as well as 2 cases of true-positive results were revealed; while in the blood GM study group (serum, $n=29$ ), 3 cases of false-positive, 4 cases of false-negative and 0 true-positive results were detected, which determines the low sensitivity of the method 
and low predictive value of a positive result of GM determination in blood, in comparison with the determination GM in BAL (Table 3).
Among the examined patients, 6 were recipients of allogeneic HSCT. They had 1 false-positive and 1 false-negative result of the study of GM in the blood and 1 false-positive result of the study of GM in the BAL (0 false-negative results).

Table 2 Results of identifying causative agents and serological markers of IA in oncological and hematological pediatric patients with focal-infiltrative changes in the lungs

\begin{tabular}{|c|c|c|c|c|}
\hline GM in BAL & GM in blood & $\begin{array}{l}\text { Positive microbiol. research } \\
\text { BAL }\end{array}$ & $\begin{array}{l}\text { Pos. microbiol. research lung } \\
\text { biopsy material }\end{array}$ & $\begin{array}{l}\text { Positive microbiol. research } \\
\text { autopsy lung material }\end{array}$ \\
\hline 0,23 & 0,15 & No & not carried out & not carried out \\
\hline 0,70 & 0,21 & No & not carried out & not carried out \\
\hline 1,00 & not explored. & No & not carried out & not carried out \\
\hline 0,22 & not explored. & No & not carried out & not carried out \\
\hline 0,48 & 0,17 & No & not carried out & not carried out \\
\hline 5,80 & 0,18 & yes/culture/Aspergillus fumigatus & not carried out & not carried out \\
\hline 0,31 & 0,19 & No & not carried out & not carried out \\
\hline $\mathrm{I}, 40$ & not explored & No & not carried out & not carried out \\
\hline 0,30 & not explored. & No & not carried out & not carried out \\
\hline 0,29 & 0,20 & No & not carried out & not carried out \\
\hline 0,29 & 0,17 & No & not carried out & not carried out \\
\hline 0,75 & 0,16 & No & not carried out & not carried out \\
\hline 1,90 & $\mathrm{I}, 20$ & No & not carried out & not carried out \\
\hline 0,35 & 0,25 & No & not carried out & not carried out \\
\hline 0,16 & 0,15 & No & yes/culture/Aspergillus flavus & not carried out \\
\hline 0,20 & $\mathrm{I}, 50$ & No & not carried out & not carried out \\
\hline 0,35 & 0,22 & No & not carried out & not carried out \\
\hline 1,00 & 0,13 & No & not carried out & not carried out \\
\hline 5,00 & 0,32 & No & not carried out & not carried out \\
\hline 0,66 & 2,00 & No & No & not carried out \\
\hline 0,28 & 0,47 & No & not carried out & not carried out \\
\hline 0,17 & 0,06 & No & not carried out & not carried out \\
\hline 0,27 & 0,07 & No & not carried out & not carried out \\
\hline 0,17 & 0,06 & No & not carried out & not carried out \\
\hline 0.13 & 0,10 & No & not carried out & No \\
\hline 1,50 & 0,20 & yes/culture/Aspergillus niger & not carried out & not carried out \\
\hline 0,22 & 0,13 & No & not carried out & yes/culture/Aspergillus spp. \\
\hline 2,00 & 0,31 & No & not carried out & not carried out \\
\hline 0,45 & 0,11 & No & not carried out & not carried out \\
\hline 0,58 & 0,20 & No & not carried out & not carried out \\
\hline 0,38 & 0,24 & No & not carried out & not carried out \\
\hline 1,30 & 0,11 & No & not carried out & not carried out \\
\hline 0,32 & 0,18 & No & not carried out & not carried out \\
\hline
\end{tabular}


Table 3 Assessment of the diagnostic value of determining GM in biological body fluids

\begin{tabular}{|c|c|c|c|c|c|}
\hline Index & $\mathbf{n}$ & $\%$ & IFI, $\mathbf{n}$ & $\%$ & $95 \% \mathrm{DI}, \%$ \\
\hline GM in BAL & 33 & 100 & 4 & 100 & - \\
\hline Positive result (index $>1.0$ ) & 9 & 27,3 & 2 & 50 & - \\
\hline Negative result (index $<1.0$ ) & 24 & 72,7 & 2 & 50 & - \\
\hline False positive result & 7 & 21,2 & - & - & - \\
\hline False negative result & 2 & 6,1 & - & - & - \\
\hline Sensitivity & - & 50 & - & - & $6,8-93,2$ \\
\hline Specificity & - & 75,9 & - & - & $56,5-89,7$ \\
\hline Negative predictive value & - & 91,7 & - & - & $80,2-96,8$ \\
\hline Positive predictive value & - & 22,2 & - & - & $8,1-48$ \\
\hline GM in blood & 29 & 100 & 4 & 100 & - \\
\hline Positive result (index $\geq 0.7$ or in two consecutive analyzes $\geq 0.5$ ) & 3 & 10,3 & 0 & 0 & - \\
\hline Negative result (index $<0.5$ ) & 26 & 89,7 & 4 & 100 & - \\
\hline False positive result & 3 & 10,3 & - & - & - \\
\hline False negative result & 4 & 13,8 & - & - & - \\
\hline Senstivity & - & 0 & - & - & $0,0-60,2$ \\
\hline Specificity & - & 88 & - & - & $68,8-97,5$ \\
\hline Negative predictive value & - & 84,6 & - & - & $82,6-86,4$ \\
\hline Positive predictive value & - & 0 & - & - & - \\
\hline
\end{tabular}

In our study, microbiological examination of BAL fluid (in all patients) and biopsy or autopsy material of lung tissue (in 4 patients) was used as a control. The severe condition of patients and the inaccessible localization of lesions limited the possibility of conducting invasive diagnostic studies to verify the diagnosis of proven Aspergillus infection in all patients.

\section{Determination of serum bis (methylthio) gliotoxin and galactomannan in children with oncological and hematological diseases}

The study included 16 patients with oncological and hematological diseases at the age from 1 to 16 years inclusive. At the time of inclusion in the study, patients had focal changes in the lungs or pathological changes in the paranasal sinuses according to computed tomography (CT) and/or clinical signs of infection (including febrile fever for 96 hours or more, despite broad-spectrum antibacterial agents). Empiric antifungal therapy was prescribed from the day the IFI was diagnosed. The definition of BMGT was carried out at the time of the diagnosis of an infectious disease before the appointment of antifungal therapy. Observation and diagnostic search in this group of patients continued.

In $1(6 \%)$ patient, the infection was subsequently proved by microbiological examination of a biopsy material of lung tissue ( $A$. nidulans), in some patients $(\mathrm{n}=9,56 \%)$ a possible invasive fungal infection was diagnosed according to the EORTC/MSG-2020 criteria, the rest patients $(n=6,38 \%)$ had a probable invasive fungal infection (due to clinical signs and a positive serum or bronchoalveolar lavage test for galactomannan). All patients with possible infection at the time of the study were in a state of neutropenia (ANC $<500 / \mathrm{pL}$ ). Half of the patients with probable infection $(n=3)$ and the patient with proven infection had normal neutrophil counts (ANC $>500 / \mathrm{pL}$ ).

All patients underwent studies of serum bis(methylthio)gliotoxin and galactomannan (before the start of antifungal therapy), and $5(31 \%)$ patients were also tested for bronchoalveolar lavage galactomannan. Among these 5 patients, 3 were positive for serum GM. The qualitative determination of bis(methylthio)gliotoxin in blood serum was carried out by the method of highly efficient chromatography-mass spectrometry. All patients ( $\mathrm{n}=16,9$ boys and 7 girls) had exposure risk factors and clinical and/or radiological signs of fungal infection.

Positive serum galactomannan was detected in 6 (37.5\%) patients, and in 1 the infection was microbiologically proven, and in one patient with probable infection, galactomannan was positive both in serum and in bronchoalveolar lavage. Galactomannan in BAL fluid was studied in 5 patients, with a positive result obtained in $2(40 \%)$ cases. In 1 patient, galactomannan was positive in both bronchoalveolar lavage and serum (as indicated above). In 1 patient with probable infection, positive galactomannan was detected only in bronchoalveolar lavage, while in serum galactomannan was negative. In 2 patients, the study of GM in BAL fluid gave a negative result, while in serum GM was positive. In 1 patient with possible infection, galactomannan was negative in both bronchoalveolar lavage and serum. Bis(methylthio) gliotoxin was not detected by the proposed method in any of the analyzed patients. The research results are presented in Table 4 .

It should be noted that only one patient who entered the study subsequently had microbiologically confirmed invasive aspergillosis, 
and the rest of the patients did not have microbiological confirmation of infection at all, which does not refute the absence of the latter in most patients. However, at the study, 4 (25\%) patients had specific changes in the lungs, characteristic of invasive aspergillosis, and recovered on the background of antifungal therapy with voriconazole.

Table 4 Characteristics of patients included in the study, the results of determining GM and BMGT

\begin{tabular}{|c|c|c|c|c|c|c|c|c|c|}
\hline № & Gender & Age, years & $\begin{array}{l}\text { Diagnosis of the } \\
\text { underlying disease }\end{array}$ & IFI & $\begin{array}{l}\text { ANC at the time of } \\
\text { the study, in } \mathrm{ml}\end{array}$ & $\begin{array}{l}\text { GM in } \\
\text { blood }\end{array}$ & $\begin{array}{l}\text { GM in } \\
\text { BAL }\end{array}$ & BMGT & $\begin{array}{l}\text { Microbiological } \\
\text { confirmation }\end{array}$ \\
\hline I & M & 15 & Brain tumor & probable & $<500$ & positive & - & negative & No \\
\hline 2 & M & 4 & ALL & probable & $<500$ & positive & - & negative & No \\
\hline 3 & $\mathrm{~F}$ & 3 & ALL & proven & $>500$ & positive & negative & negative & Yes \\
\hline 4 & M & 12 & AML & possible & $<500$ & negative & - & negative & No \\
\hline 5 & M & 2 & $\begin{array}{l}\text { primary } \\
\text { immunodeficiency }\end{array}$ & probable & $>500$ & positive & - & negative & No \\
\hline 6 & $\mathrm{~F}$ & 5 & AML & possible & $<500$ & negative & - & negative & No \\
\hline 7 & $M$ & 10 & AML & possible & $<500$ & negative & - & negative & No \\
\hline 8 & $\mathrm{~F}$ & 6 & AML & possible & $<500$ & negative & negative & negative & No \\
\hline 9 & M & 12 & $\begin{array}{l}\text { MDS, condition after } \\
\text { haploHSCT }\end{array}$ & possible & $<500$ & negative & - & negative & No \\
\hline 10 & $\mathrm{~F}$ & 16 & AML & probable & $>500$ & negative & positive & negative & No \\
\hline II & M & 16 & AML & possible & $<500$ & negative & - & negative & No \\
\hline 12 & $M$ & 10 & Lymphoma & possible & $<500$ & negative & - & negative & No \\
\hline 13 & $M$ & II & AML & possible & $<500$ & negative & - & negative & No \\
\hline 14 & $\mathrm{~F}$ & I & RMS & probable & $>500$ & positive & negative & negative & No \\
\hline 15 & $\mathrm{~F}$ & 7 & $\mathrm{AML}$ & possible & $<500$ & negative & - & negative & No \\
\hline 16 & $\mathrm{~F}$ & 7 & AML & probable & $<500$ & positive & positive & negative & No \\
\hline
\end{tabular}

\section{Discussion}

The limitation of the study of PCT and CRP in the differential diagnosis of fungal and bacterial infections was the relatively small number of patients, although the sample size was sufficient to achieve statistical significance in the analysis. The results are comparable to those in adults. There are publications on an increased level of CRP and a low level of PCT in adult hematological patients with fungal infection in the study of the etiology of febrile neutropenia. ${ }^{41}$ The levels of PCT and CRP have not been studied in patients without signs of infection. This study design was chosen due to the need to distinguish etiological factors of infectious complications in oncology and hematology.

Histological or microbiological examination of lung tissue obtained by thoracoscopy or thoracotomy is mandatory in the diagnosis of invasive pulmonary aspergillosis. ${ }^{42}$ Microbiological testing, including microscopy and culture, remains the gold standard for laboratory diagnosis of fungal infections. However, due to the risk of bleeding or other complications, lung biopsy is not always possible during an invasive diagnostic test. At the same time, the presence of clinical or radiological markers of IFI in the presence of negative results of microbiological studies forces an immediate start of expensive and toxic therapy. That is why invasive serological tests for the determination of fungal antigens are an additional option in the diagnosis of mycoses. When using them, it should be taken into account the possibility of obtaining false positive and false negative results. For this reason, according to the latest guidelines for the management of febrile neutropenia in cancer patients and pediatric HSCT recipients (USA, 2017), if fever persists $>96 \mathrm{~h}$, despite the use of broad-spectrum antibiotics, it is not necessary to use a serum galactomannan study for the purpose of diagnostics of fungal infection..$^{43}$ At the same time, BAL is included in the diagnostic procedures for identifying the etiology of infectious lesions of the lower respiratory tract. Performing BAL helps the clinician make a diagnosis, find out the etiology of the infectious lesion, and promptly initiate etiotropic treatment.

The diagnosis of invasive aspergillosis is difficult, the clinical manifestations are often nonspecific, and the final diagnosis depends on invasive procedures, which are not always feasible due to the severe condition of the patient. A sensitive and specific test is required for early diagnosis of this life-threatening complication and rational antifungal treatment in order to improve the effectiveness of therapy, reduce toxicity and reduce costs. We determined the biomarker bis(methylthio)gliotoxin in the serum of children with oncological and hematological diseases, with clinical, serological and radiological signs of fungal infection (invasive aspergillosis). BMGT was not detected in serum by HPLC-MS in any of the analyzed patients.

A limitation of this study is the small number of patients. Moreover, in most of the analyzed patients, invasive mycosis was not microbiologically confirmed. However, 3 out of 6 patients with probable IFI and 1 patient with proven IFI had focal changes in the lungs characteristic of invasive aspergillosis, and recovered on the antifungal therapy, which may indirectly indicate the presence 
of invasive aspergillosis in them. It should also be mentioned that the method for determining BMGT using HPLC-MS is laborious; moreover, not all hospitals have the necessary equipment.

\section{Conclusion}

An increase in the level of C-reactive protein with a normal level of procalcitonin in immunocompromised children with clinical signs of bloodstream infection indicates a fungal etiology of the disease. With fungal infections of the bloodstream in children with oncological and hematological diseases, the level of procalcitonin during an infectious episode is significantly lower than with bacterial infections of the bloodstream $(p=0.0063)$, and the level of C-reactive protein exceeds the norm, but does not have significant differences in the comparison groups $(\mathrm{p}=0.1719)$. Determination of the optical density index of galactomannan in bronchoalveolar fluid is a sensitive marker for the diagnosis of invasive pulmonary aspergillosis. Diagnostic study of galactomannan in bronchoalveolar lavage in children has a high predictive value of a negative result (91.7\%). The sensitivity of the method is higher than in the study of galactomannan in blood serum ( $50 \%$ versus $0 \%)$. There was no correlation between serum bis (methylthio) gliotoxin levels and the presence of invasive aspergillosis in children.

\section{Acknowledgments}

None.

\section{Conflicts of interest}

Authors declare that there is no conflict of interest.

\section{References}

1. Morell M, Fraser VJ, Kollef MH. Delaying the empiric treatment of candida bloodstream infection until positive blood culture results are obtained a potential risk factor for hospital mortality. Antimicrobial Agents Chemotherapy. 2005;49(9):3640-3645.

2. Pennisi M, Antonelli M. Clinical aspects of invasive candidiasis in critically ill patients. Drugs. 2009;69(suppl 1):21-28.

3. Reichenberger $F$, et al. Lung resection for invasive pulmonary aspergillosis in neutropenic patients with hematologic diseases. Am J Respir Crit Care Medicine. 1998;158(3):885-890.

4. Upton A, Kirby KA, Paul C, et al. Invasive aspergillosis following hematopoietic cell transplantation: outcomes and prognostic factors associated with mortality. Clinical Infectious Diseases. 2007;44(4):531540.

5. Hope WW, Kruhlak MJ, Lyman CA, et al. Pathogenesis of Aspergillus fumigatus and the kinetics of galactomannan in an in vitro model of early invasive pulmonary aspergillosis: implications for antifungal therapy. $J$ Infect Dis. 2007;195(3):455-466.

6. Maertens J, Buvé K, Theunissen K, et al. Galactomannan serves as a surrogate endpoint for outcome of pulmonary invasive aspergillosis in neutropenic hematology patients. Cancer. 2009;115(2):355-362.

7. Clancy CJ, Nguyen MH. Finding the "missing 50\%" of invasive candidiasis: How nonculture diagnostics will improve understanding of disease spectrum and transform patient care. Clin Infect Dis. 2013;56(9):1284-1292.

8. Cornely OA. Galactomannan testing during mold-active prophylaxis. Clin Infect Dis. 2014;59(12):1703-1704.

9. Peter Donnelly J, Chen SC, Kauffman CA, et al. Revision and Update of the Consensus Definitions of Invasive Fungal Disease From the European Organization for Research and Treatment of Cancer and the Mycoses Study Group Education and Research Consortium. Clinical Infectious Diseases. 2020;71(6):1367-1376.
10. Arvanitis M, Anagnostou T, Fuchs BB et al. Molecular and nonmolecular diagnostic methods for invasive fungal infections. Clin Microbiol Rev. 2014;27(3):490-526.

11. Zhang SX. Enhancing molecular approaches for diagnosis of fungal infections. Future Microbiol. 2013;8(12):1599-1611.

12. Riedel S, Eisinger SW, Dam L, et al. Comparison of BD Bactec Plus Aerobic/F medium to VersaTREK Redox 1 blood culture medium for detection of Candida spp. in seeded blood culture specimens containing therapeutic levels of antifungal agents. J Clin Microbiol. 2011;49(4):15241529 .

13. Reinhart K, Meisner M. Biomarkers in the critically ill patient: procalcitonin. Crit Care Clin. 2011;27(2):253-263.

14. Igor Stoma, Igor Karpov, Anatoly Uss, et al. Low Levels of Procalcitonin or Presepsin Combined with Significantly Elevated C-reactive Protein May Suggest an Invasive Fungal Infection in Hematological Patients With Febrile Neutropenia. Hemasphere. 2019;3(1):e170.

15. Jha AK, Bansal D, Chakrabarti A, et al. Serum galactomannan assay for the diagnosis of invasive aspergillosis in children with haematological malignancies. Mycoses. 2013;56(4):442-4484.

16. Chong GM, Maertens JA, Driessen GJ, et al. Diagnostic performance of galactomannan antigen testing in cerebrospinal fluid. $J$ Clinical Microbiology. 2016;54(2):428-431.

17. Duettmann W, Koidl C, Troppan K, et al. Serum and urine galactomannan testing for screening in patients with hematological malignancies. Med Mycology. 2014;52(6):647-652.

18. Duettmann W, Koidl C, Krause R, et al. Specificity of mannan antigen and anti-mannan antibody screening in patients with haematological malignancies at risk for fungal infection. Mycoses. 2016;59(6):374-378.

19. Duarte RF, Sanchez-Ortega I, Cuesta I, et al. Serum galactomannanbased early detection of invasive aspergillosis in hematology patients receiving effective antimold prophylaxis. Clinical Infectious Diseases. 2014;59(12):1696-1702.

20. Martín-Rabadán P, Gijon P, Roberto AF, et al. False-positive Aspergillus antigenemia due to blood product conditioning fluids. Clinical Infectious Diseases. 2012;55(4):e22-e27.

21. Aubry A, Porcher R, Julie B, et al. Occurrence and kinetics of falsepositive Aspergillus galactomannan test results following treatment with beta-lactam antibiotics in patients with hematological disorders. $J$ Clinical Microbiology. 2006;44(2):389-394.

22. Asano-Mori Y, Kanda Y, Kumi O, et al. False-positive Aspergillus galactomannan antigenaemia after haematopoietic stem cell transplantation. J Antimicrobial Chemotherapy. 2008;61(2):411-416.

23. Mennink-Kersten MA, Donnelly JP, Verweij PE. Detection of circulating galactomannan for the diagnosis and management of invasive aspergillosis. Lancet Infectious Diseases. 2004;4(6):349-357.

24. Tortorano AM, Esposto MC, Anna P, et al. Cross-reactivity of Fusarium spp. in the Aspergillus Galactomannan enzyme-linked immunosorbent assay. J Clinical Microbiology. 2012;50(3):1051-1053.

25. Huang YT, Hung CC, Hsueh PR. Aspergillus galactomannan antigenemia in penicilliosis marneffei. AIDS. 2007;21(14):1990-1991.

26. Wheat LJ, Emily H, Durkin M, et al. Histoplasmosis-associated crossreactivity in the BioRad Platelia Aspergillus enzyme immunoassay. Clinical Vaccine Immunology. 2007;14(5):638-640.

27. Papanikolaou I, Stenger R, Bessot JC, et al. Anaphylactic shock to guar gum (food additive E412) contained in a meal substitute. Allergy. 2007;62(7):822

28. J Springer, M Lackner, D Nachbaur, et al. Prospective multicentre PCRbased Aspergillus DNA screening in high-risk patients with and without primary antifungal mould prophylaxis. Clinical Microbiology Infection. 2016;22(1):80-86. 
29. Johan Maertens, Johan Van Eldere, Jan Verhaegen, et al. Use of circulating galactomannan screening for early diagnosis of invasive aspergillosis in allogeneic stem cell transplant recipients. $J$ Infectious Diseases. 2002;186(9):1297-1306.

30. Johan Maertens, Koen Theunissen, Eric Verbeken, et al. Prospective clinical evaluation of lower cut-offs for galactomannan detection in adult neutropenic cancer patients and haematological stem cell transplant recipients. Brit J Haematology. 2004;126(6):852-860.

31. Arvanitis M, Mylonakis E. Diagnosis of invasive aspergillosis: recent developments and ongoing challenges. Eur $J$ Clinical Investigation. 2015;45(6):646-652.

32. Bondy GS, Pestka JJ. Immunomodulation by fungal toxins. $J$ Toxicology Environmental Health Part B Crit Rev. 2000;3(2):109-143.

33. Russell E Lewis, Nathan P Wiederhold, Jingduan Chi, et al. Detection of gliotoxin in experimental and human aspergillosis. Infection a Immunity. 2005;73(1):635-637.

34. Claudio Kupfahl, Anna Michalka, Cornelia Lass-Flörl, et al. Gliotoxin production by clinical and environmental Aspergillus fumigatus strains. Intern J of Med Microbiology. 2007;298(3/4):319-327.

35. Janyce A Sugui, Julian Pardo, Yun C Chang, et al. Gliotoxin is a virulence factor of Aspergillus fumigatus: gliP deletion attenuates virulence in mice immunosuppressed with hydrocortisone. Eukaryotic Cell. 2007;6(9):1562-1569.

36. Claudio Kupfahl, Thomas Ruppert, Annebärbel Dietz, et al. Candida species fail to produce the immunosuppressive secondary metabolite gliotoxin in vitro. FEMS Yeast Research. 2007;7(6):986-992.
37. Maria P Domingo, Cristina Colmenarejo, Luis Martínez-Lostao, et al. Bis(methyl)gliotoxin proves to be a more stable and reliable marker for invasive aspergillosis than gliotoxin and suitable for use in diagnosis. Diagnostic Microbiology Infectious Disease. 2012;73(1):57-64.

38. Manuel Cuenca-Estrella, Matteo Bassetti, Cornelia Lass-Flörl, et al. Detection and investigation of invasive mould disease. $J$ Antimicrobial Chemotherapy. 2011;66(Suppl 1):i15-i24.

39. Vidal-Garcia M, Domingo MP, Rueda B, et al. Clinical validity of bis(methylthio)gliotoxin for the diagnosis of invasive aspergillosis. Appl Microbiology Biotechnology. 2016;100(5):2327-2334.

40. Mercaldo ND. Confidence intervals for predictive values with an emphasis to case-control studies. Statistics in Medicine. 2007;26(10):2170-2183.

41. Stoma I, Karpov I, Uss A, et al. Low levels of procalcitonin or presepsin combined with significantly elevated c-reactive protein may suggest an invasive fungal infection in hematological patients with febrile neutropenia. HemaSphere. 2019;3(1):e170.

42. Juergen Prattes, Holger Flick, Florian Prüller, et al. Novel tests for diagnosis of invasive aspergillosis in patients with underlying respiratory diseases. Am J Respiratory Crit Care Medicine. 2014;190(8):922-929.

43. Thomas Lehrnbecher, Paula Robinson, Brian Fisher, et al. Guideline for the management of fever and neutropenia in children with cancer and hematopoietic stem-cell transplantation recipients: 2017 update. $J$ Clinical Oncology. 2017;35(18):2082-2094. 